\title{
MENINGOCELE, GLIOMA Y MENINGIOMA DEL NERVIO ÓPTICO: DIAGNÓSTICO DIFERENCIAL Y TRATAMIENTO
}

\author{
MENINGOCELE, GLIOMA AND OPTIC NERVE MENINGIOMA: \\ DIFFERENTIAL DIAGNOSIS AND TREATMENT
}

\author{
GENOL ${ }^{1}$, TROYANO J ${ }^{1}$, ARIÑO $\mathrm{M}^{1}$, IGLESIAS I ${ }^{1}$, ARRIOLA P ${ }^{1}$, GARCÍA-SÁNCHEZ J ${ }^{2}$
}

\begin{abstract}
RESUMEN
Objetivo: Estudiar a partir de 3 casos las características clínicas y radiológicas del meningocele, meningioma y glioma del nervio óptico (NO) para discutir su diagnóstico diferencial así como su enfoque terapéutico.

Método: Se revisaron las historias clínicas de tres pacientes afectos de las patologías anteriormente mencionadas que fueron estudiados en nuestro servicio, y se realizó una revisión bibliográfica sobre el diagnóstico y tratamiento actual de estas entidades.

Resultados: El diagnóstico diferencial debe basarse en un amplio abanico de aspectos: edad, raza, sexo y frecuencia de la tumoración como factores epidemiológicos a tener en cuenta. Clínicamente los pilares fundamentales son: agudeza visual (AV), campimetría, exoftalmometría Hertel y funduscopia, que nos servirán para controlar la evolución del proceso. Las características radiológicas que nos aportan la resonancia magnética $(\mathrm{RM})$ y la tomografía computerizada (TC) como técnicas de elección nos ayudan a diferenciar un cuadro de otro. En raras ocasiones hay que recurrir al estudio anatomopatológico para confirmar la entidad. Las opciones terapéuticas son observación, cirugía, quimioterapia y radioterapia en función de las características del caso.
\end{abstract}

\begin{abstract}
Purpose: After studying 3 clinical cases, we have reviewed the clinical and radiological characteristics of meningocele, meningioma and optic nerve glioma. The differential diagnosis and therapeutic management are also discussed.

Methods: Review of three clinical reports of three patients seen in our unit and a bibliographic search concerning the diagnosis and therapeutic management of these three entities at the present time.

Results: Differential diagnosis has to be based on a wide range of parameters: epidemiologic (age, race, sex, prevalence of the tumors), clinical (visual acuity, perimetry, Hertel exophthalmometry and funduscopy) and radiologic (computed tomography and magnetic resonance). Anatomopathologic study is required only rarely. The therapeutic options are: observation, surgery and radiotherapy. Conclusion: A correct differential diagnosis is mandatory to be able to individualize the treatment for each entity (Arch Soc Esp Oftalmol 2009; 84: 563-568).
\end{abstract}

Key words: Meningioma, meningocele, glioma, optic nerve.

\footnotetext{
Recibido: 31/5/07. Aceptado: 24/11/09.

Departamento de Cirugía Oculoplástica y Órbita. Hospital Clínico San Carlos. Madrid. España.

${ }^{1}$ Licenciado en Medicina.

2 Doctor en Medicina. Especialista en Oftalmología Universidad Complutense de Madrid.

Comunicación presentada en el LXXXII Congreso de la S.E.O. (La Coruña 2006).

Correspondencia:

Ignacio Genol Saavedra

C/. Piamonte, $7,3 .^{\circ} \mathrm{B}$

28004 Madrid

España

E-mail: ignaciogenol@gmail.com
} 
Conclusión: El diagnóstico diferencial de estas patologías es difícil, y hay que basarse en sus características clínicas y radiológicas para así orientar correctamente su tratamiento, individualizando cada caso y teniendo en cuenta el comportamiento relativamente benigno de estos tumores para evitar una decisión terapéutica precipitada.

Palabras clave: Meningioma, meningocele, glioma, nervio óptico.

\section{INTRODUCCIÓN}

La patología quirúrgica del nervio óptico (NO) presenta ciertas peculiaridades caracterizadas por su enclave anatómico, que dificulta su accesibilidad. El riesgo de invasión intracraneal y el deterioro en la función visual nos obliga a tipificar cada tipo de lesión. El meningocele del NO, también descrito en la literatura como quiste de la aracnoides o ectasia de las vainas del nervio óptico, es una proliferación de tejido fibrovascular normal que provoca una distensión benigna de las meninges. Estos quistes pueden simular neoplasias del NO, en especial meningiomas. Los meningiomas primarios del NO suponen un tercio de los tumores primarios del NO y son las neoplasias más frecuentes de entre las que interesan a las vainas del NO. Los gliomas del NO a pesar de ser poco frecuentes, son la causa más frecuente de neoplasia del NO.

\section{SUJETOS, MATERIAL Y MÉTODOS}

A partir de tres casos planteamos las características clínicas y radiológicas de un meningocele, un meningioma y un glioma del NO para discutir su diagnóstico diferencial así como su tratamiento apoyados en los últimos avances encontrados en la literatura.

\section{RESULTADOS}

\section{Caso 1}

Paciente varón de 39 años que acude al Servicio de Urgencias por presentar proptosis de un año de evolución en ambos ojos (AO), sin descenso de la agudeza visual (AV). No presentaba ningún otro síntoma a destacar ni antecedentes de interés. En la exploración el paciente presentaba una AV de 0,5 en ojo derecho (OD) (referido como amblíope por el paciente) y de 0,8 en ojo izquierdo (OI). La exoftalmometría Hertel era de $25 \mathrm{~mm}$ en OD y de 23 $\mathrm{mm}$ en OI. La exploración biomicroscópica y tonométrica fue normal. En el estudio del fondo de ojo presentaba edema de papila en OD. El test de colores Farnsworth era normal. En el estudio campimétrico el OI era normal pero en el OD presentaba un defecto inferonasal, que no era una cuadrantanopsia estrictamente. Se solicitó una tomografía computerizada (TC) que se informó como ectasia/engrosamiento de las vainas de ambos $\mathrm{NO}$ en contexto de exoftalmos bilateral más marcado en OD (fig. 1). Sospechándose la presencia de meningocele bilateral se optó por observar periódicamente al paciente sin realizar ningún otro acto diagnóstico-terapéutico adicional. En la actualidad, 16 años después, el

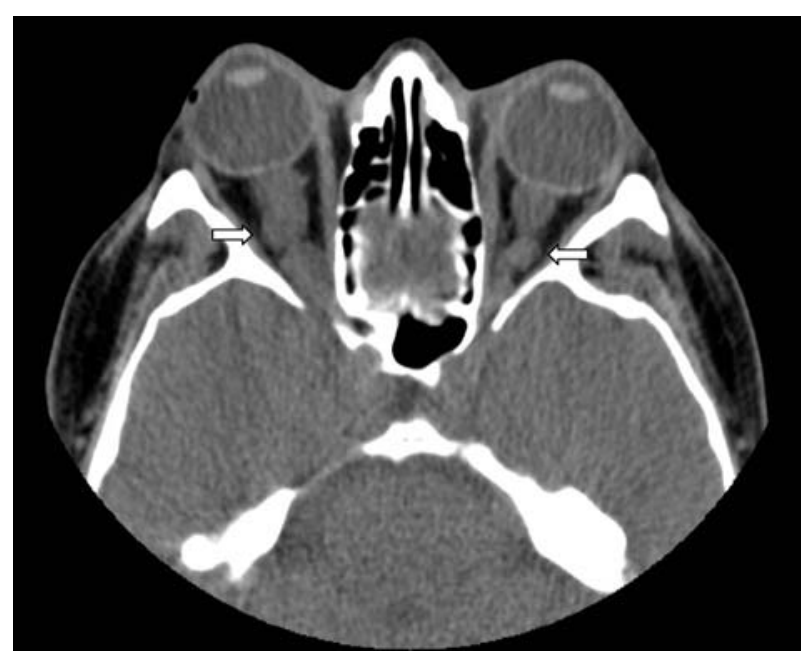

Fig. 1: Meningocele bilateral (flechas). Tomografía computerizada axial. 
paciente presenta una AV de 0,6 en OD y 0,8 en OI. La exoftalmometría y campimetría han permanecido estables. En el fondo de ojo el aspecto papilar apenas ha variado. Continúa con sus revisiones periódicas.

\section{Caso 2}

Mujer de 38 años remitida desde un hospital comarcal a nuestro centro para valoración de tumoración de NO. En su historia clínica cabe destacar un descenso de la $\mathrm{AV}$ progresivo en el último año que se exacerbó en los últimos 2 ó 3 meses. En julio 2005 su AV era de 0,7, en agosto de 0,5, en octubre de 0,1 , en noviembre de cuenta dedos a un metro y en diciembre del 2005 cuando nos fue remitida presentaba amaurosis en OD y defecto pupilar aferente absoluto. La exoftalmometría no reflejaba asimetrías entre AO. Desde el primer momento en que se estudió a la paciente presentó un edema de papila en OD con normalidad de OI. La TC fue informada como asimetría en los nervios ópticos estando el derecho levemente engrosado en relación con el nervio contralateral. Se procedió a hacer una resonancia magnética nuclear (RMN) orbitaria en diciembre de 2005 que se informó como engrosamiento difuso y concéntrico de la vaina dural del NO derecho, con ausencia de realce del propio nervio y que se extiende a lo largo de todo su trayecto intraorbitario en conducto óptico y extensión intracraneal alcanzando clinoides anterior derecha, sospechándose un meningioma del NO derecho (fig. 2). Tras explicar las opciones terapéuticas, la paciente rechaza cualquier tipo de actuación y actualmente sigue revisiones periódicas.

\section{Caso 3}

Varón de 3 años que estaba siendo estudiado por neurólogos por sospecha de neurofibromatosis tipo 1 ya que cumplía al menos un criterio diagnóstico mayor (manchas café con leche diseminadas). Presentaba exoftalmos axial OD desde hace 5 meses. Su AV con test de Pigassou era de 1. Se realizó una RMN que se informó como engrosamiento bien delimitado, homogéneo e isodenso del NO con meninges intactas compatible con el diagnóstico de glioma del $\mathrm{NO}$ en su porción intracanalicular e intraorbitaria (fig. 3) confirmándose así el diagnós-

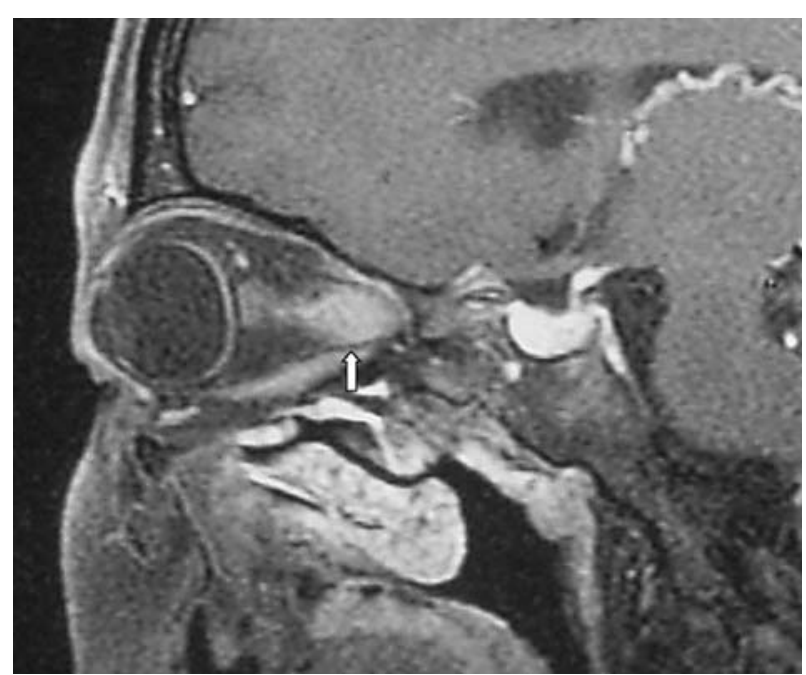

Fig. 2: Meningioma de las vainas del nervio óptico (NO) derecho. Obsérvese la ausencia de realce en la resonancia magnética nuclear del propio NO (flecha) en un corte tangencial.

tico de neurofibromatosis tipo 1 pues cumplía dos criterios diagnósticos mayores. Dado que no presentaba lagoftalmos y su AV estaba conservada se

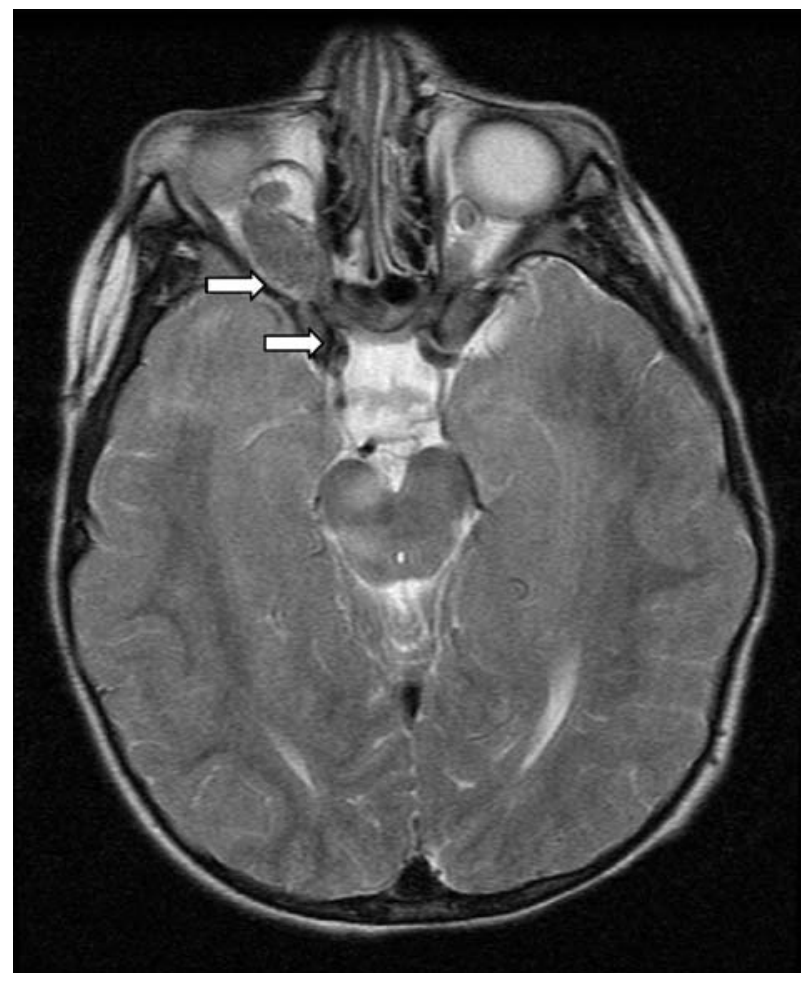

Fig. 3: Resonancia magnética nuclear axial. Glioma intraorbitario e intracanalicular (flechas). 
optó por revisiones cada 3 meses durante el primer año, y controles cada 6 meses durante el segundo año. Aunque los criterios de progresión de los gliomas ópticos no están universalmente definidos, en este caso 4 años después de su diagnóstico, tanto la AV como el tamaño radiológico de la lesión se mantienen estables, por lo que de momento no planteamos tratamiento quimioterápico y controlamos al paciente cada 6 meses, realizándose RMN anualmente.

\section{DISCUSIÓN}

El diagnóstico diferencial entre estas patologías no siempre es fácil y debe fundamentarse en un abordaje desde una perspectiva multifactorial. Los datos epidemiológicos, la presentación y evolución clínica y las características radiológicas de la lesión son datos suficientes para diagnosticar estas patologías en la mayor parte de los casos. Excepcionalmente se requieren estudios anatomopatológicos para definir estas entidades. Desde el punto de vista epidemiológico, los meningoceles son entidades excepcionales. La única revisión de casos en la literatura encontrada (1) hace referencia a 12 quistes aracnoideos, por tanto es muy difícil saber su distribución sexual y en qué edad de la vida son más frecuentes. Los meningiomas son el tumor de vainas del NO más frecuente, típico de mujeres entre 30 y 50 años y suponen un $2 \%$ de los tumores orbitarios (2). Los gliomas son la neoplasia más frecuente del NO apareciendo la mayoría de ellos $(85 \%)$ en menores de 20 años y especialmente en menores de 10 años (65\%). Se asocian típicamente a neurofibromatosis tipo 1 (Enfermedad de Von Recklinghausen) en torno a un $25 \%$, en cuyo caso suelen ser multifocales y/o bilaterales (3), por lo que incluso se consideran como uno de los seis criterios diagnósticos de esta entidad. El 15\% de los pacientes con neurofibromatosis tipo 1 presentan un glioma de NO (4). La clínica nos ayuda a orientar cada caso centrándonos especialmente en la información que nos aporta la AV, el aspecto del fondo de ojo y las características de la proptosis. En cuanto a los cambios en la $\mathrm{AV}$, en los pacientes con meningocele no suele verse afectada dado que apenas se comprime el NO (1), en los gliomas es típico el debut con discromatopsia y descenso muy lento de la AV (5) (aunque ésta a veces es difícil valorar pues suelen presentarse en edades tempranas). En los meningio- mas el descenso en la AV es precoz y evoluciona con mayor rapidez (2). Respecto al aspecto funduscópico el quiste de aracnoides puede producir edema de papila que suele mantenerse en un mismo estadío de gravedad en toda la evolución. En los meningiomas, donde también se presenta el edema de papila, este progresa conforme evoluciona el tumor, pero lo más típico son los shunts optociliares. En los gliomas, la mayoría presentan cierto grado de atrofia óptica y también suelen asociar edema de papila especialmente aquellos que se limitan al NO y no al quiasma o porciones más elevadas de las vías ópticas.

Una vez, tenemos la sospecha clínica es obligado recurrir a los estudios de imagen de alta resolución, TC o RMN. En la TC de un quiste aracnoideo puede presentar una imagen lobulada o quística, pero con NO indemne y sin calcificaciones intralesionales (1). En un meningioma la captación de contraste suele ser homogénea. El signo de «vía de tren», originalmente descrito en estos tumores cuando se observan las vainas del NO engrosadas y densas delineando un centro lúcido, puede observarse también en otras patologías, como linfomas o incluso pseudotumores orbitarios periópticos. Lo más típico de los meningiomas en un TC son las calcificaciones y sobre todo que éstos rodean al NO y lo comprimen atenuando su diámetro (2), contrastando con la imagen de los gliomas, en los que el NO aparece expandido con aspecto tortuoso, con meninges intactas y presentando rara vez calcificaciones (6).

Si tras un estudio clínico y radiológico el cuadro aún presentara dudas diagnósticas, lo cual es excepcional, podremos recurrir al estudio histopatológico de estas lesiones. Las ectasias de las vainas del NO son simples proliferaciones de células meningoepiteliales que adoptan una forma quística macroscópica que no ofrece dudas (1). La mayoría de los meningiomas orbitarios al abordarlos vemos como se extienden a través de la duramadre, lo cual no ocurre en los gliomas, y en su estudio anatomopatológico observamos como la proliferación de células de las capas externas de la aracnoides se organizan en anillos que pueden tener cierto grado de degeneración hialina y típicamente calcificarse formando los clásicos cuerpos de psammoma (7). En los gliomas, la proliferación primaria es a expensas de astrocitos, que pueden estimular una proliferación fibrovascular de células meningoepiteliales aracnoideas que se dispongan formando cuerpos de psammoma, pero en este caso excepcionalmente se calcifican (8). 
Una vez tipificado el cuadro, debemos plantearnos el adecuado abordaje terapéutico de cada caso clínico. Los meningoceles, cuyo origen puede ser congénito, idiopático o postraumático no suelen precisar tratamiento alguno $(1,9)$. Debemos hacer un seguimiento estrecho del paciente pues hay casos descritos (10) de progresión del edema de papila o de descenso de la AV que nos obligan a plantearnos intervenir terapéuticamente. Inicialmente se puede plantear la opción de tratar al paciente con inhibidores de la anhidrasa carbónica sistémicos basándonos en la posibilidad de que el gradiente osmótico entre el espacio subaracnoideo cerebral y el perióptico provoque una disminución en el tamaño de la ectasia. En los casos de meningoceles sintomáticos, el papel de los corticoides intravenosos no ha sido discutido en las publicaciones existentes. La punción y aspiración de estos quistes, se reserva especialmente para los casos postraumáticos donde la disfunción del $\mathrm{NO}$ es manifiesta y la recuperación del edema de papila así como la mejora de la AV tiene mayores posibilidades de producirse.

En el manejo de los meningiomas siempre debemos plantearnos: ¿necesita tratamiento?, ¿puede esperar a ser tratado el tumor? A pesar de presentar un porcentaje muy bajo de metástasis y mortalidad, actualmente se recomienda tratar a los pacientes por varios motivos $(2,11,12,13,14,15)$ : la progresión en la pérdida de AV ocurre en el $85 \%$ de los casos y hasta en el 38\% de los casos con afectación intracanalicular se han descrito alteraciones en el ojo contralateral. Además observar al paciente y no tratar el tumor mientras aumenta su tamaño y progresa el deterioro visual, podría disminuir las probabilidades de éxito de una posible futura intervención terapéutica. En pacientes menores de 20 años se presume que el comportamiento de los meningiomas es más agresivo por lo que la opción de observar sin tratar debe adoptarse con prudencia individualizando cada caso.

Los casos seleccionados para observar sin tratar serían aquellos que mantienen una buena AV y aquellos que no presentan morbilidad asociada (proptosis...) realizándose controles oftalmológicos, radiológicos y neurológicos anualmente, o cada 6 meses si el paciente es menor de 20 años.

En los casos seleccionados para tratar, es decir aquellos meningiomas que progresivamente empeoran la $\mathrm{AV}$ del paciente o que provocan exoftalmos severo o que tienen riesgo de expansión intracraneal, disponemos de dos armas terapéuticas: cirugía de la neoplasia y radioterapia. La cirugía de extirpación de un meningioma primario del NO está asociada a un alto riesgo de ceguera y en los casos en los que quedasen masas de tumor residual éstas tienen un alto potencial de malignizarse, por tanto esta opción debería reservarse sólo para aquellos casos de neoplasias primarias de localización anterior, con crecimiento exofítico e infiltración clara de la duramadre focalmente. Es excepcional que pacientes con deterioro visual se puedan beneficiar de la fenestración del tumor, incluso podría argumentarse que en algunos casos este procedimiento se ha asociado a infiltración masiva de la órbita por parte del tumor (2), y aunque sí es posible que en los casos en los que el tumor se ve distendido por líquido cefalorraquídeo atrapado entre sus vainas, la fenestración pueda frenar el deterioro visual, en la actualidad su práctica está abandonada (16), así como la realización de biopsias por su alta comorbilidad (17).

La radioterapia inicialmente se usó como adyuvante a la cirugía, pero en el 2002 Turbin demostró en una serie retrospectiva de 64 casos (14) que la radioterapia fraccionada estereotáctica como único tratamiento de los meningiomas primarios del NO es la opción terapéutica que mejores resultados visuales ofrece (el 80\% mantienen o mejoran su función visual) y por tanto se considera como el tratamiento de elección en aquellos meningiomas que requieran ser tratados $(12,18)$. A pesar de esto algunos autores (incluido el propio Turbin) defienden la utilidad de la cirugía como medida adyuvante a la radioterapia en casos seleccionados $(19,20)$.

El tratamiento de los gliomas (3) es el más controvertido, y siempre dependerá de la localización del tumor, su velocidad de crecimiento y del tamaño y síntomas que produzca. Aún no se ha alcanzado un consenso universal sobre los criterios a emplear para definir si el glioma está progresando o no (siendo los más empleados la evidencia del aumento del tamaño en RMN y/o pérdida de agudeza visual). Cuando se limita al NO el pronóstico de vida es excelente, y si conservan una AV útil, el manejo conservador y el seguimiento puntual de la enfermedad es lo más indicado. Por tanto sólo se debe tratar cuando la progresión del tumor es indudable. En la actualidad la quimioterapia es el tratamiento de elección, siendo la combinación de vincristina y carboplatino la más recomendada (21). La radioterapia sólo se reserva para los casos en los que no han funcionado las combinaciones quimio- 
terápicas, dado que tienen un alto riesgo de complicaciones mayores (endocrinopatías y accidentes cerebrovasculares). Afortunadamente las técnicas de radiación cada vez son más selectivas y actúan sobre el foco tumoral con un mínimo efecto sobre las estructuras adyacentes (radioterapia estereotáctica fraccionada y la radioterapia guiada por gamma knife), pero todavía es una opción terapéutica de segunda línea pues el riesgo de transformación maligna del glioma tras la radioterapia persiste.

La opción quirúrgica sólo se plantea en los casos de tumor confinado al NO con márgenes limpios en el contexto de paciente con ceguera, exoftalmos severo o cuando la extensión a través del segmento intracraneal amenaza el quiasma. Si hay afectación quiasmática, el riesgo de muerte por compromiso hipotalámico o del tercer ventrículo aumenta al $28 \%$, y en estos casos la cirugía esta asociada a un alto índice de morbimortalidad y no parece aumentar la supervivencia, prefiriéndose optar por la radioterapia.

\section{BIBLIOGRAFÍA}

1 Akor C, Wojno TH, Newman NJ, Grossniklaus HE. Arachnoid cyst of the optic nerve: report of two cases and review of the literature. Ophthal Plast Reconstr Surg 2003; 19: 466-469.

2. Eddleman CS, Liu JK. Optic nerve sheath meningioma: current diagnosis and treatment. Neurosurg Focus 2007; 23: E4.

3. Liu GT. Optic gliomas of the anterior visual pathway. Curr Opin Ophthalmol 2006; 17: 427-431.

4. North K. Neurofibromatosis type 1: review of the first 200 patients in an Australian clinic. J Child Neurol 1993; 8. 395-402.

5. Thiagalingham S, Flaherty M, Bilson F, North K. Neurofibromatosis type 1 and optic pathway gliomas. Ophthalmology 2004; 111: 568-577.

6. Pérez-Moreiras JV. Glioma del nervio óptico. In: PérezMoreiras JV. Patología orbitaria. Barcelona: Edika Med; 2002; II: 649-682.
7. Pérez-Moreiras JV. Meningiomas del nervio óptico. In: Pérez-Moreiras JV. Patología orbitaria. Barcelona: Edika Med; 2002; II: 683-723.

8. Rootman J. Optic nerve glioma. Pilocytic (juvenile) astrocytoma. In: Rootman J. Diseases of the orbit. A multidisciplinary approach. Philadelphia: Lippincott Williams \& Wilkins; 2003; 213-216.

9. Jungkim S, Khurshid SG, Fenton S. Dural ectasia of the optic nerve sheath. Acta Ophthalmol Scand 2005; 83: 266-267.

10. Mesa-Gutiérrez JC, Quiñones SM, Ginebreda JA. Optic nerve sheath meningocele. Clin Ophthalmol 2008; 2: 661664.

11. Berman D, Miller NR. New concepts in the management of optic nerve sheath meningiomas. Ann Acad Med Singapore 2006; 35: 168-174.

12. Dutton JJ. Optic nerve sheath meningiomas. Surv Ophthalmol 1992; 37: 167-183.

13. Kennerdell JS, Maroon JC, Malton M, Warren FA. The management of optic nerve sheath meningiomas. Am J Ophthalmol 1988; 106: 450-457.

14. Turbin RE, Thompson CR, Kennerdell JS, Cockerham KP, Kupersmith MJ. A long-term visual outcome comparison in patients with optic nerve sheath meningioma managed with observation, surgery, radiotherapy, or surgery and radiotherapy. Ophthalmology 2002; 109: 890-899.

15. Harold Lee HB, Garrity JA, Cameron JD, Strianese D, Bonavolontá G, Patrinely JR. Primary optic nerve sheath meningioma in children. Surv Ophthalmol 2008; 53: 543558.

16. Carrasco JR, Penne RB. Optic nerve sheath meningiomas and advanced treatment options. Curr Opin Ophthalmol 2004; 15: 406-410.

17. Kim JW, Rizzo JF, Lessell S. Controversies in the management of optic nerve sheath meningiomas. Int Ophthalmol Clin 2005; 45: 5-23.

18. Pitz, S, Becker G, Schiefer U, Wilhelm H, Jeremic B, Bamberg $M$, et al. Stereotactic fractionated irradiation of optic nerve sheath meningioma: a new treatment alternative. $\mathrm{Br}$ J Ophthalmol 2002; 86:1265-1268.

19. Turbin RE, Wladis EJ, Frohman LP, Langer PD, Kennerdell JS. Role for surgery as adjuvant therapy in optic nerve sheath meningioma. Ophthal Plast Reconstr Surg 2006; 22: 278-282.

20. Turbin RE, Pokorny K. Diagnosis and treatment of orbital optic nerve sheath meningioma. Cancer Control 2004; 11: 334-341.

21. Jahraus CD, Tarbell NJ. Optic pathway gliomas. Pediatr Blood Cancer 2006; 46: 586-596. 